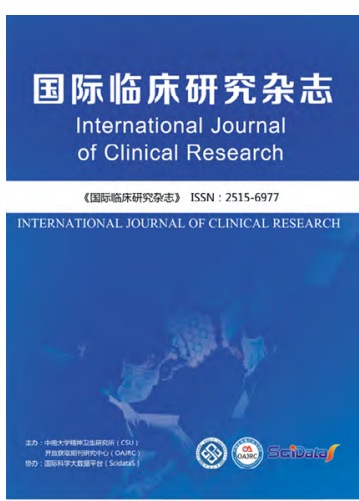

http://ijcr.oajrc.org

GOPEN ACCESS

DOI: $10.12208 /$ j.ijcr.20190005

收稿日期：2019-06-18

出刊日期: 2019-07-09

通讯作者: 马娜, 中南大学湘雅医院, 长沙
术后早期量化活动方案在腹腔镜肝切除患者术后加 速康复中的应用

周蓝, 马娜, 卜冬梅, 陶静, 龚连生

中南大学湘雅医院，长沙

摘要

目的：研究探讨术后早期量化活动方案在腹腔镜肝切除患者 术后加速康复中的应用效果。

方法：选取 2017 年 6 月 2018 年 12 月在本院接受腹腔镜肝 切除患者 96 例为研究对象, 随机分为观察组和对照组, 各 48 例。 对照组患者围术期常规护理干预，观察组在此基础上增加术后早 期量化活动方案干预。对两组患者的术后恢复指标、下床活动情况、 疼痛评分、睡眠质量、并发症发生率、等进行评估对比。

结果：观察组患者首次排气时间、开始下床活动时间、住院 时间、住院费用均低于对照组患者, 组间比较差异有统计学意义 $(P<0.05)$ 。观察组在术后第 $1 \mathrm{~d}$ 、第 $2 \mathrm{~d}$ 、第 $3 \mathrm{~d}$ 活动步数、活动 距离均高于对照组患者, 组间比较差异有统计学意义 $(P<0.05)$ 。 观察组在术后第 $1 d$ 、第 $2 d$ 、第 $3 d$ 疼痛评分低于对照组, 睡眠 时间高于对照组，组间差异明显 $(P<0.05)$ 。观察组并发症发生率 为 $6.25 \%(4 / 48)$, 低于对照组患者为 $16.67 \%(8 / 48)$, 组间差异显著 $(P<0.05)$ 。

结论: 术后早期量化活动方案在腹腔镜肝切除患者术后加速 康复中的应用效果良好, 能够加速患者术后的康复进程, 能指导 患者术后正确安排活动并增加活动量，改善睡眠治疗，降低并发 症发生率。

关键词：腹腔镜肝切除术; 加速康复; 早期量化活动方案; 活动量

\section{Application of Early Quantitative Activity Program in Accelerated Rehabilitation after Laparoscopic Hepatectomy}

Lan Zhou, Na Ma, Dongmei Bu, Jing Tao, Liansheng Gong

Xiangya hospital of central south university, Changsha 


\begin{abstract}
Objective: To study the effect of early quantitative activity program in accelerated rehabilitation after laparoscopic hepatectomy.

Methods: 96 patients who underwent laparoscopic hepatectomy in our hospital from June 2017 to December 2018 were randomly divided into observation group and control group, 48 cases in each group. Patients in the control group received routine nursing intervention during perioperative period, while patients in the observation group received early quantitative activity program intervention after operation. The two groups were evaluated and compared in terms of recovery index, out-of-bed activity, pain score, sleep quality, incidence of complications and so on.
\end{abstract}

Results: The first exhaust time, the time of getting out of bed, the time of hospitalization and the cost of hospitalization in the observation group were lower than those in the control group, and the difference between the two groups was statistically significant $(P<0.05)$. The activity steps, distance and time of the observation group on the 1st, 2nd and 3rd day after operation were higher than those of the control group, and there was significant difference between the two groups $(P<0.05)$. The pain scores of the observation group on the 1st, 2nd and 3rd day after operation were lower than those of the control group, and the sleep time was higher than that of the control group. The difference between the two groups was significant $(P<.05)$. The incidence of complications in the observation group was $8.33 \%$ (4/48), which was lower than that in the control group $(25.00 \%$ $(12 / 48)$. There was a significant difference between the two groups $(P<0.05)$.

Conclusion: Quantitative activity program in early stage after laparoscopic hepatectomy has good effect in accelerating the recovery of patients after laparoscopic hepatectomy. It can accelerate the recovery process of patients after laparoscopic hepatectomy, increase the activity of patients after operation, improve sleep therapy and reduce the incidence of complications.

Key words: Laparoscopic hepatectomy; Accelerated rehabilitation; Early quantitative activity program; Activity

原发性肝癌是临床常见的恶性肿瘤疾病, 具 有较高的发病率和病死率，严重威胁到患者的生 命健康。目前临床上对于原发性肝癌的治疗多采 用手术切除的办法切除病灶对患者的病情予以缓 解 [1]。常规的开腹病灶切除术由于手术创伤性 较大，术中失血量多，术后并发症发生率高，不 利于患者的预后，对手术效果产生不良影响。而 近些年发展起来的腹腔镜下肝癌切除术充分发挥 了腔镜技术和微创手术的优点, 相比于常规开腹 手术, 极大的减轻了患者的痛苦, 也缩短了患者
术后的康复进程，目前已逐渐成为原发性肝癌手 术治疗的首选方法 [2]。虽然相比于开放式手术 而言，腹腔镜肝切除术的创伤性显著降低，但患 者仍然存在着术后应激性问题，患者免疫功能降 低, 术后易出现并发症等影响患者的术后康复进 程和手术效果 [1-2]。在腹腔镜肝切除患者围术期 采取适宜的护理干预措施能够改善患者的预后。 近些年随着加速理论实践的快速发展, 康复外科 中的快速康复护理理念在临床外科手术中广为推 崇，是以循证医学为基础在患者围术期开展的一 系列的护理干预措施, 以减少患者在手术过程中 的应激反应，促进患者术后的快速康复，减少围术 期不良事件的发生 [3-4]。已有相关报道证实，患 者术后进行早期的量化活动干预，可以促进患者 身体机能的恢复, 减少并发症的发生，缩短患者的 康复进程 [5-6]。为此, 在本研究中以本院近期收 治的原发性肝癌行腹腔镜肝切除患者为研究对象, 对其实施术后早期量化活动方案干预，观察评估 对患者加速康复的影响。

\section{1. 资料与方法}

\section{1 临床资料}

选取 2017 年 6 月 2018 年 12 月在本院接受 腹腔镜肝切除患者 96 例为研究对象, 纳入标准 [7]: (1) 患者经诊断均为原发性肝癌患者; (2) 患 者均具有腹腔镜下肝切除手术治疗的指征; (3) 患 者精神状态良好, 能够配合治疗和护理干预过程 的进行实施; (4) 患者签署知情同意书, 研究方案 经医院伦理学委员会批准。排除标准: (1)年龄 $<18$ 或大于 65 岁 (2)有严重心肺疾病、凝血功能障碍 等不能耐受手术者; (3)术前影像学检查提示肿瘤 已有远处转移者; (4)任何原因致无法配合本研究 患者, 如: 语言理解障碍、电话通讯障碍等。剔 除标准: (1)伴发其他非肿瘤性疾病导致患者不能 执行该量化活动方案; (2)术中探查或术后病理证 实有远处转移; (3)术中失血量 $>1500 \mathrm{ml}$ 。上述患 者按随机分为观察组和对照组, 各 48 例。观察 
组: 男性 28 例、女性 20 例, 年龄 29 62 岁, 平 均年龄 (45.98 \pm 9.23$)$ 岁, 美国麻醉协会 (American Anesthesia Association, ASA) 分 级: I 级 25 例、II 级 23 例, 肝功能 Child-Pugh 分级: A 级 29 例、B 级 19 例; 对照组: 男性 24 例、女性 24 例, 年龄 $33 \sim 65$ 岁, 平均年龄 $(47.19 \pm 8.78)$ 岁, ASA 分级为: I 级 27 例、II 级 21 例, 肝功能 Child-Pugh 分级: $\mathrm{A}$ 级 30 例、 $\mathrm{B}$ 级 18 例; 两组患者一般资料比较无 统计学意义 $(P>0.05)$ 。

\section{2 治疗方法}

对照组患者围术期常规护理干预, 患者进行 术前的健康宣教和心理疏导, 减轻患者对手术的 恐惧感; 术中进行严密的心电监护, 根据患者在 术中表现出现应激状态调整麻醉药的给药方案,

并做好输液管理; 术后对患者进行常规的体征监 测、用药指导、饮食指导和运动训练指导、出院 指导等。

观察组在对照组基础上增加术后早期量化 活动方案干预, 主要内容包括: (1) 成立术后早 期量化活动干预小组, 小组成员由本科室的医护 人员组成, 本科室护士长担任小组的组长, 全面 负责术后早期量化活动干预。(2) 护理小组成员 通过查阅文献资料和病例资料，针对患者的自身 情况并结合手术方案制定患者的术后早期量化活 动方案, 并对每一位管床护士进行统一规范化培 训, 以充分保障早期活动指导的同质性。(3) 由 责任护士在术前向患者及家属详细宣教术后活动 量的安排并指导患者掌握正确进行握拳运动、踝 泵运动的方法, 责任护士根据术后早期量化活动 方案的内容干预完成每日术后活动量。(4) 术后 当天, 患者麻醉清醒送回至病房后即适当抬高床 头 $15^{\circ} \sim 30^{\circ}$, 由护士或家属协助患者床上翻 身, 每隔 $2 \mathrm{~h}$ 进行 1 次; 期间对患者的下肢肌肉 进行按摩, 促进下肢血液循环; 患者一般情况好 转后, 指导患者进行床上的主动活动, 双手握拳 运动及踝泵运动, 反复 20 30 次为一组, 每隔 $1 \mathrm{~h}$ 进行 5 10 组。(5) 术后第 $1 \mathrm{~d}$, 可抬高患者床
头 $45^{\circ} \sim 90^{\circ}$ ，协助患者进行缓慢的坐起、翻身 运动, 并指导患者主动的关节功能恢复训练, 主 要包括髋关节、膝关节、时关节、肩关节、踝关 节等, 每次活动 $10 \mathrm{~min} \sim 15 \mathrm{~min}$, 每隔 2 3h 进行 一次, 为患者的下床活动做准备。晨查房时, 经 医护共同评估后，停床旁监护仪，由责任护士协 助完成首次下床活动, 患者沿着床旁进行站立和 缓慢短距离步行活动, 步行活动时间 5 10 min, 术后 $1 \mathrm{~d}$ 完成 2 次下床活动, 患者下床活动量不 应超过患者的最大耐受力。(6) 术后第 $2 \mathrm{~d}$, 由责 任护士或家属协助患者进行更大量的下床活动, 换扶患者在病房内进行行走训练, 行走训练时间 $15 \sim 20 \mathrm{~min} /$ 次, 3 次 $/ \mathrm{d}$, 对于恢复情况较好的患 者可不需借助家属、护士的撸扶, 独立进行步行 训练。期间协助患者自主进行洗脸、刷牙、穿衣 服等日常活动。(7) 术后第 $3 \mathrm{~d}$, 患者在助行器的 帮助下, 进行自主的病房外走廊间的较长距离的 步行行走活动, 每次 20 30min, 3 5 次 /d, 根据 患者自身耐受力情况进行酌情的增量或减量, 下 床活动时间不低于 3 个小时。期间患者可自主完 成进食、如则等日常活动。8患者在术毕回病房 后至术后 3 天佩戴运动手环记录活动及睡眠情况, 研究者每日 18：00 于患者床前观察患者 18：00 前活动的完成情况, 未完成部分督促患者夜间睡 觉前继续完成, 次日上午查房前, 查看并记录前 1 天活动反馈信息 [8]。

\section{3 观察指标}

对两组患者术后临床指标排气时间、开始下 床活动时间、术后住院时间、住院费用等进行统 计对比。对患者术后第 $1 d$ 、第 $2 d$ 、第 $3 d$ 的活动 步数、步行距离、疼痛评分、夜间睡眠时间进行 测量对比。患者活动步数、活动距离及睡眠情况 通过运动手环进行监测, 疼痛评分以视觉模拟评 分法 (Visual analogue scoring, VAS) 进行评估, 该 评分量表满分为 10 分, 得分越高表明患者的疼 痛程度越严重。最后, 对两组患者术后并发症发 生率进行统计对比。 


\section{4 统计学处理}

以 SPSS 20.0 进行数据处理与分析, 计量资 料以 $(\bar{x} \pm \mathrm{s})$ 表示, 组间对比实施 $\mathrm{t}$ 检验, 计数资 料以 $[\mathrm{n}(\%)]$ 表示, 组间对比实施 $x^{2}$ 检验, 当 $P$ $<0.05$ 时差异有统计学意义。

表 1 术后临床指标对比 $(\bar{x} \pm s)$

\section{2. 结果}

\section{1 术后临床指标对比}

观察组患者首次排气时间、开始下床活动时 间、术后住院时间、住院费用均低于对照组患者, 组间比较差异有统计学意义 $(P<0.05)$, 见表 1 。

\begin{tabular}{cccccc}
\hline 组别 & 例数 & $\begin{array}{c}\text { 首次排气 } \\
\text { 时间 }(\mathrm{h})\end{array}$ & $\begin{array}{c}\text { 首次下床活 } \\
\text { 动时间 }(\mathrm{h})\end{array}$ & $\begin{array}{c}\text { 术后住院时间 } \\
(\mathrm{d})\end{array}$ & $\begin{array}{c}\text { 住院费用 } \\
(\text { 元 })\end{array}$ \\
\hline 观察组 & 48 & $18.28 \pm 6.34$ & $15.34 \pm 4.01$ & $5.12 \pm 0.84$ & $31156.76 \pm 8091.46$ \\
对照组 & 48 & $33.66 \pm 6.68$ & $32.45 \pm 6.35$ & $7.08 \pm 1.12$ & $38535.42 \pm 9606.58$ \\
$t$ & - & 11.570 & 15.784 & 9.699 & 4.070 \\
$P$ & - & 0.000 & 0.014 & 0.000 & 0.000 \\
\hline
\end{tabular}

\section{2 术后活动量指标对比}

观察组在术后第 $1 d$ 、第 $2 \mathrm{~d}$ 、第 $3 \mathrm{~d}$ 活动步数、 步行距离均高于对照组患者, 组间比较差异有统 计学意义 $(P<0.05)$, 见表 2 。
2.3 术后疼痛评分、睡眠时间对比

观察组在术后第 $1 d$ 、第 $2 d$ 、第 $3 d$ 疼痛评分 低于对照组，睡眠时间高于对照组，组间差异明 显 $(P<0.05)$ ，见表 3 。

表 2 术后活动量指标对比 $(\bar{x} \pm s)$

\begin{tabular}{|c|c|c|c|c|c|c|c|}
\hline \multirow{2}{*}{ 组别 } & \multirow{2}{*}{ 例数 } & \multicolumn{3}{|c|}{ 活动步数 ( 步 ) } & \multicolumn{3}{|c|}{ 步行距离 (m) } \\
\hline & & 第 $1 \mathrm{~d}$ & 第 $2 \mathrm{~d}$ & 第 $3 d$ & 第 1d & 第 $2 \mathrm{~d}$ & 第 3d \\
\hline $\begin{array}{c}\text { 观察 } \\
\text { 组 }\end{array}$ & 48 & $195.19 \pm 48.08$ & $552.41 \pm 146.35$ & $980.30 \pm 104.34$ & $115.75 \pm 10.35$ & $226.44 \pm 28.82$ & $319.73 \pm 62.57$ \\
\hline $\begin{array}{c}\text { 对照 } \\
\text { 组 }\end{array}$ & 48 & $83.71 \pm 45.34$ & $289.76 \pm 120.41$ & $619.73 \pm 198.23$ & $51.34 \pm 9.83$ & $121.47 \pm 30.59$ & $258.83 \pm 70.08$ \\
\hline $\mathrm{t}$ & - & 11.687 & 9.602 & 11.152 & 31.262 & 17.304 & 4.491 \\
\hline $\mathrm{P}$ & - & 0.000 & 0.000 & 0.000 & 0.000 & 0.000 & 0.010 \\
\hline
\end{tabular}

表 3 术后疼痛评分、睡眠时间对比 $(\bar{x} \pm s)$

\begin{tabular}{ccccccccc}
\hline \multirow{2}{*}{ 组别 } & \multirow{2}{*}{ 例数 } & \multicolumn{3}{c}{ VAS 评分 $($ 分 $)$} & & \multicolumn{3}{c}{ 睡眠时间 $(\mathrm{h})$} \\
\cline { 3 - 5 } \cline { 6 - 8 } 第 $1 \mathrm{~d}$ & 第 $2 \mathrm{~d}$ & 第 $3 \mathrm{~d}$ & & 第 $2 \mathrm{~d}$ & 第 $3 \mathrm{~d}$ \\
\hline 观察组 & 48 & $3.45 \pm 1.08$ & $1.45 \pm 0.67$ & $0.87 \pm 0.34$ & & $6.18 \pm 1.67$ & $6.89 \pm 1.56$ & $7.48 \pm 1.49$ \\
对照组 & 48 & $4.21 \pm 1.21$ & $3.01 \pm 0.72$ & $1.19 \pm 0.42$ & & $5.41 \pm 1.49$ & $6.20 \pm 1.52$ & $6.67 \pm 1.51$ \\
$\mathrm{t}$ & - & 3.246 & 11.071 & 4.103 & & 2.384 & 2.195 & 2.645 \\
$\mathrm{P}$ & - & 0.002 & 0.000 & 0.000 & & 0.019 & 0.031 & 0.010 \\
\hline
\end{tabular}


2.4 并发症发生率对比

观察组并发症发生率为 $6.25 \%(4 / 48)$, 低 $\quad(P<0.05)$, 见表 4 。

表 4 并发症发生率比较 $[\mathrm{n}(\%)]$
于对照组患者为 $16.67 \%(8 / 48)$, 组间差异显著$$
\text { (P<0.05), 见表4。 }
$$

\begin{tabular}{cccccccc}
\hline 组别 & 例数 & 切口愈合不良 & 肺部感染 & 腹腔积液 & 切口出血 & 深静脉血栓 & 总发生率 \\
\hline 观察组 & 48 & $1(2.08)$ & $1(2.08)$ & $1(2.08)$ & $0(0.00)$ & $0(0.00)$ & $3(6.25)$ \\
对照组 & 48 & $2(4.17)$ & $2(4.17)$ & $2(4.17)$ & $1(2.08)$ & $1(2.08)$ & $8(16.67)$ \\
$x^{2}$ & & & & & & & 4.566 \\
$P$ & & & & & & & 0.039 \\
\hline
\end{tabular}

\section{3. 讨论}

快速康复护理理念最早于 21 世纪初有欧洲 学者提出, 是建立在患者生理、病理变化的基础 上, 以循证医学为依据, 以减少患者治疗期间不 必要的应激反应和外源性的损伤, 从而可为患者 病情的顺利治疗提供保障 [9]。目前, 国外在加 速康复外科领域中的理论和实践研究相对成熟, 并逐步应用于各种外科手术的术后康复中。在国 内已有大量文献表明加速康复优于常规的外科治 疗, 但如何确保进一步规范和优化并执行加速康 复的相关策略仍需探讨。术后早期活动干预是加 速康复的重要内容, 可以促进肌肉、呼吸系统、 胃肠道功能恢复, 减少术后血栓栓塞、胰岛素抵 抗的发生, 能够缩短患者的术后康复进程 [10]。 但由于不正确的观念存在, 部分患者及家属误认 为术后早期活动会引起伤口裂开、伤口疼痛, 导 致患者在术后早期不敢进行主动活动, 肝癌患者 由于肝功能不良、凝血功能差、手术创伤大、并 发症多, 患者术后常卧床 $3 \sim 4 \mathrm{~d}$ 才开始下床活动。 专家共识提出: 腹腔镜肝切除术后第一天可下床 活动, 应制定个体化的活动计划, 鼓励早期活动。 但目前缺乏腹腔镜肝切除患者早期下床活动及其 影响的循证依据, 缺乏可操作、易量化的早期下 床活动方案来指导患者术后的活动 [11]。在本研 究中, 通过对患者治疗个体化的科学有效的术后 早期量化活动方案, 并对患者进行有目的、有计
划的早期活动干预，从而有效加快了患者术后的 康复进程。

患者术后首次排气时间、开始下床活动时间、 住院时间、住院费用等均是反映术后康复进程快 慢的重要指标, 观察组患者的上述指标水平均低 于对照组, 表明术后早期量化活动方案的实施能 够加快患者的术后恢复进程，佐证了既往的临床 报道中的研究结果 [12]。这是因为通过术后早期 量化活动方案的实施, 改善患者肠麻痹, 促进胃 肠功能恢复和身体机能的恢复, 并且患者术后当 天回归病房后即开展身体各关节组织的被动运 动, 为患者下床活动做好了充足的准备工作, 因 此患者术后恢复时间和住院时间大大缩短 [13]。 术后的活动量是评价患者的术后加速康复的重要 指标, 观察组经早期量化活动方案干预, 患者术 后 $3 \mathrm{~d}$ 内的活动步数、步行距离均高于对照组, 这是因为观察组中对于患者在术后当天至术后的 $3 \mathrm{~d}$ 内, 制定了科学系统的运动计划, 将活动量大 小由依次递增的顺序合理安排在术后 $3 \mathrm{~d}$ 内完成, 合理分配运动时间, 患者的依从性提高, 运动量 也显著增加, 这与临床既往报道的结果基本相似 [14-15]。另外, 在本研究中随着患者的运动量的 增加, 患者术后的疼痛感、睡眠时间等指标均有 显著的改善, 分析其原因可能是因为患者运动量 的增加, 转移患者注意力, 增强患者康复的信心, 
有利于缓解患者的疼痛, 并且患者的运动量增加 使得患者产生轻微的疲㤂感, 更有利于促进患者 的睡眠 [16]。并发症的发生也是评价患者术后康 复效果好坏的一项关键指标, 观察组患者加强了 术后的早期量化活动干预，促进患者各项机能的 恢复, 使血液循环、组织功能趋于正常, 避免了 静脉血栓、伤口感染等并发症的发生, 因而观察 组并发症发生率低于对照组, 验证了刘冰心等人 关于此方面的研究结论 [17]。

综上所述, 术后早期量化活动方案在腹腔镜 肝切除患者术后加速康复中的应用效果良好, 能 够加速患者术后的康复进程, 提高患者的术后早 期下床活动的依从性以及增加术后活动量, 改善 睡眠治疗, 降低并发症发生率。本研究样本量较 少, 病种单一, 在今后的 ERAS 临床护理实践中, 应推进医护一体化不断整合有循证医学证据的研 究结果, 进一步探讨如何确保规范和优化 ERAS 相关策略的执行，促进患者全面快速康复。

\section{参考文献}

[1] 骆鹏飞, 荚卫东, 许戈良, 等. 加速康复外 科理念在原发性肝癌患者肝切除围手术 期中的应用 [J]. 中华普通外科杂志, 2015, 30(11): 862-865.

[2]雷秋成, 王新颖, 郑华珍, 等. 加速康复外 科在腹腔镜肝切除术应用安全性与有效性 的 Meta 分析 [J]. 中国普通外科杂志, 2014, 23(7): 946-952.

[3] Terasawa M, Ishizawa $T$, Mise $Y$, et al. Applications of fusion-fluorescence imaging using indocyanine green in laparoscopic hepatectomy [J]. Surgical Endoscopy, 2017, 31(1): 1-8.

[4] 韩冰, 丁义涛, 朱新华, 等. 加速康复外科理 念在腹腔镜肝切除术中的应用 [J]. 临床肝胆 病杂志, 2018, 34(3): 120-123.
[5]夏灿灿, 江志伟, 王刚, 等. 胃肠道肿瘤术后 患者早期下床活动的量化研究及护理效果 [J]. 医学研究生学报, 2016, 29(4): 411-415.

[6] 刘连新, 陈亚进, 曹铭辉, 等. 腹腔镜肝切除 术加速康复外科中国专家共识 (2017版) [J]. 中国实用外科杂志, 2017, 29(5): 61-68.

[7] 王龙金金, 赵战强, 谭宏涛, 等. 加速康复外科 在肝癌肝切除术中的临床应用研究 [J]. 国际 外科学杂志, 2016, 43(4): 249-254.

[8]夏灿灿, 江志伟, 王刚, 冯啸波, 杨洋, 叶 向红, 黄迎春, 彭南海. 胃肠道肿瘤术后患 者早期下床活动的量化研究及护理效果 [J]. 医学研究生学报, 2016, 29(4): 411-415.

[9] Lwin T M, Hoffman R M, Bouvet M. Regarding the applications of fusion-fluorescence imaging using indocyanine green in laparoscopic hepatectomy [J]. Translational Gastroenterology \& Hepatology, 2017, 2(9): 70-76.

[10] Xu X, Chen J, Wang F, et al. Recurrence of Hepatocellular Carcinoma After Laparoscopic Hepatectomy: Risk Factors and Treatment Strategies [J]. Journal of Laparoendoscopic \& Advanced Surgical Techniques Part A, 2017, 27(7): 676-681.

[11] 中华医学会外科学分会外科手术学学组, 中 国医疗保健国际交流促进会, 加速康复外科 学分会肝脏外科学组. 肝切除术后加速康复 中国专家共识 (2017 版 ) [J]. 中华肝脏外科 手术学电子杂志 ,2017, 6(4): 254-260.

[12] Komatsu S, Brustia R, Goumard C, et al. Clinical impact of laparoscopic hepatectomy: technical and oncological viewpoints [J]. Surgical Endoscopy, 2017, 31(3): 1442-1450.

[13] 李佳, 刘怡素. 术后早期活动在肝癌肝叶切 除患者术后加速康复中的应用 $[\mathrm{J}]$. 中华现 
代护理杂志, 2017, 23(6): 781-783.

[14] 麦结珍, 李巧云, 温文敏. 加速康复外科在 腹腔镜肝癌切除术围手术期康复护理的应 用 [J]. 消化肿瘤杂志: 电子版, 2015, 7(4): 223-226.

[15] Lim C, Osseis M, Lahat E, et al. Safety of laparoscopic hepatectomy in patients with hepatocellular carcinoma and portal hypertension: interim analysis of an open prospective study
[J]. Surgical Endoscopy, 2019, 33(3): 811-820.

[16] Jian-Hong, Zhong, Ning-Fu, et al. Is laparoscopic hepatectomy superior to open hepatectomy for hepatocellular carcinoma $[\mathrm{J}]$. World Journal of Hepatology, 2017, 9(4): 167-170.

[17] 刘冰心 , 郭婷. 胃癌手术患者快速康复外科 护理中量化活动方案的实施 [J]. 护理学杂 志, 2018, 33(10): 27-30. 